\title{
Theoretical and Experimental Analyses of Tensor-Based Regression and Classification
}

\author{
Kishan Wimalawarne \\ Department of Computer Science \\ Tokyo Institute of Technology \\ 2-12-1 Ookayama, Meguro-ku, \\ Tokyo 152-8552, Japan. \\ kishanwn@gmail.com \\ Ryota Tomioka \\ The Toyota Technological Institute at Chicago, \\ 6045 S. Kenwood Av., \\ Chicago, IL 60637, USA. \\ tomioka@ttic.edu \\ Masashi Sugiyama \\ Department of Complexity Science and Engineering \\ University of Tokyo \\ 7-3-1 Hongo, Bunkyo-ku, \\ Tokyo 113-0033, Japan. \\ sugi@k.u-tokyo.ac.jp
}

\begin{abstract}
We theoretically and experimentally investigate tensor-based regression and classification. Our focus is regularization with various tensor norms, including the overlapped trace norm, the latent trace norm, and the scaled latent trace norm. We first give dual optimization methods using the alternating direction method of multipliers, which is computationally efficient when the number of training samples is moderate. We then theoretically derive an excess risk bound for each tensor norm and clarify their behavior. Finally, we perform extensive experiments using simulated and real data and demonstrate the superiority of tensor-based learning methods over vector- and matrix-based learning methods.
\end{abstract}

\section{Introduction}

A wide range of real-world data takes the format of matrices and tensors, e.g., recommendation (Karatzoglou et al., 2010), video sequences (Kim et al., 2007), climates 
(Bahadori et al., 2014), genomes (Sankaranarayanan et al., 2015), and neuro-imaging (Zhou et al., 2013). A naive way to learn from such matrix and tensor data is to vectorize them and apply ordinary regression or classification methods designed for vectorial data. However, such a vectorization approach would lead to loss in structural information of matrices and tensors such as low-rankness.

The objective of this paper is to investigate regression and classification methods that directly handle tensor data without vectorization. Low-rank structure of data has been successfully utilized in various applications such as missing data imputation (Cai et al., 2010), robust principal component analysis (Candès et al., 2011), and subspace clustering (Liu et al., 2010). In this paper, instead of low-rankness of data itself, we consider its dual-learning coefficients of a regressor and a classifier. Low-rankness in learning coefficients means that only a subspace of feature space is used for regression and classification.

For matrices, regression and classification has been studied in Tomioka and Aihara (2007) and Zhou and Li (2014) in the context of EEG data analysis. It was experimentally demonstrated that directly learning matrix data by low-rank regularization can significantly improve the performance compared to learning after vectorization. Another advantage of using low-rank regularization in the context of EEG data analysis is that analyzing singular value spectra of learning coefficients is useful in understanding activities of brain regions.

More recently, an inductive learning method for tensors has been explored (Signoretto et al., 2013). Compared to the matrix case, learning with tensors is inherently more complex. For example, the multilinear ranks of tensors make it more complicated to find a proper low-rankness of a tensor compared to matrices which has only one rank. So far, several tensor norms such as the overlapped trace norm or the tensor nuclear norm (Liu et al., 2009), the latent trace norm (Tomioka and Suzuki, 2013), and the scaled latent trace norm (Wimalawarne et al., 2014) have been proposed and demonstrated to perform well for various tensor structures. However, theoretical analysis of tensor learning in inductive learning settings has not been much investigated yet. Another challenge in inductive tensor learning is efficient optimization strategies, since tensor data often has much higher dimensionalities than matrix and vector data.

In this paper, we theoretically and experimentally investigate tensor-based regression and classification with regularization by the overlapped trace norm, the latent trace norm, and the scaled latent trace norm. We first provide their dual formulations and propose optimization procedures using the alternating direction method of multipliers (Bertsekas, 1996), which is computationally efficient when the number of data samples is moderate. We then derive an excess risk bound for each tensor regularization, which allows us to theoretically understand the behavior of tensor norm regularization. More specifically, we elucidate that the excess risk of the overlapped trace norm is bounded with the average multilinear ranks of each mode, that of the latent trace norm is bounded with the minimum multilinear rank among all modes, and that of the scaled latent trace norm is bounded with the minimum ratio between multilinear ranks and mode dimensions. Finally, for simulated and real tensor data, we experimentally investigate the behavior of tensor-based regression and classification methods. The experimental results are in concordance with our theoretical findings, and tensor-based learning methods compare favorably with vector- and matrix-based methods. 
The remainder of the paper is organized as follows. In Section 2, we formulate the problem of tensor-based supervised learning and review the overlapped trace norm, the latent trace norm, and the scaled latent trace norm. In Section 3, we derive dual optimization algorithms based on the alternating direction method of multipliers. In Section 4, we theoretically give an excess risk bound for each tensor norm. In Section 5, we give experimental results on both artificial and real-world data and illustrate the advantage of tensor-based learning methods. Finally, in Section 6, we conclude this paper.

\section{Notation}

Throughout the paper, we use standard tensor notation following Kolda and Bader (2009). We represent a $K$-way tensor as $\mathcal{W} \in \mathbb{R}^{n_{1} \times \cdots \times n_{K}}$ that consists of $N=\prod_{k=1}^{K} n_{k}$ elements. A mode- $k$ fiber of $\mathcal{W}$ is an $n_{k}$-dimensional vector which can be obtained by fixing all except the $k$ th index. The mode- $k$ unfolding of tensor $\mathcal{W}$ is represented as $W_{(k)} \in \mathbb{R}^{n_{k} \times N / n_{k}}$ which is obtained by concatenating all the $N / n_{k}$ mode- $k$ fibers along its columns. The spectral norm of a matrix $X$ is denoted by $\|X\|_{\text {op }}$ which is the maximum singular value of $X$. The operator $\langle\mathcal{W}, \mathcal{X}\rangle$ is the sum of element-wise multiplications of $\mathcal{W}$ and $\mathcal{X}$, i.e., $\langle\mathcal{W}, \mathcal{X}\rangle=\operatorname{vec}(\mathcal{W})^{\top} \operatorname{vec}(\mathcal{X})$. The Frobenius norm of a tensor $\mathcal{X}$ is defined as $\|\mathcal{X}\|_{\mathrm{F}}=\sqrt{\langle\mathcal{X}, \mathcal{X}\rangle}$

\section{Learning with Tensor Regularization}

In this section, we put forward inductive tensor learning models with tensor regularization and review different tensor norms used for low-rank regularization.

\subsection{Problem Formulation}

Our focus in this paper is regression and classification of tensor data. Let us consider a data set $\left(\mathcal{X}_{i}, y_{i}\right), i=1, \ldots, m$, where $\mathcal{X}_{i} \in \mathbb{R}^{n_{1} \times \cdots \times n_{K}}$ is a covariate tensor and $y_{i}$ is a target. $y_{i} \in \mathbb{R}$ for regression, while $y_{i} \in\{-1,1\}$ for classification. We consider the following learning model for a tensor norm $\|\cdot\|_{\star}$ :

$$
\min _{\mathcal{W}, b} \sum_{i=1}^{m} l\left(\mathcal{X}_{i}, y_{i}, \mathcal{W}, b\right)+\lambda\|\mathcal{W}\|_{\star},
$$

where $l\left(\mathcal{X}_{i}, y_{i}, \mathcal{W}, b\right)$ is the loss function: the squared loss,

$$
l\left(\mathcal{X}_{i}, y_{i}, \mathcal{W}, b\right)=\left(y_{i}-\left(\left\langle\mathcal{W}, \mathcal{X}_{i}\right\rangle+b\right)\right)^{2}
$$

is used for regression, and the logistic loss,

$$
l\left(\mathcal{X}_{i}, y_{i}, \mathcal{W}, b\right)=\log \left(1+\exp \left(-y_{i}\left(\left\langle\mathcal{W}, \mathcal{X}_{i}\right\rangle+b\right)\right)\right.
$$

is used for classification. $b \in \mathbb{R}$ is the bias term and $\lambda \geq 0$ is the regularization parameter. If $\|\cdot\|_{\star}=\|\cdot\|_{2}$ or $\|\cdot\|_{1}$, then the above problem is equivalent to ordinary vector-based $l_{2^{-}}$or $l_{1}$-regularization. 
To understand the effect of tensor-based regularization, it is important to investigate the low-rankness of tensors. When considering a matrix $W \in \mathbb{R}^{n_{1} \times n_{2}}$, its trace norm is defined as

$$
\|W\|_{\text {tr }}=\sum_{j=1}^{J} \sigma_{j},
$$

where $\sigma_{j}$ is the $j^{\text {th }}$ singular value and $J$ is the number of non-zero singular values $(J \leq$ $\left.\min \left(n_{1}, n_{2}\right)\right)$. A matrix is called law rank if $J<\min \left(n_{1}, n_{2}\right)$. The matrix trace norm (4) is a convex envelop to the matrix rank and it is commonly used in matrix low-rank approximation (Recht et al., 2010).

As in matrices, the rank property is also available for tensors, but it is more complicated due to its multidimensional structure. The mode- $k$ rank $r_{k}$ of a tensor $\mathcal{W} \in \mathbb{R}^{n_{1} \times \cdots \times n_{K}}$ is defined as the rank of mode- $k$ unfolding $W_{(k)}$ and the multilinear rank of $\mathcal{W}$ is given as $\left(r_{1}, \ldots, r_{K}\right)$. The mode- $i$ of a tensor $\mathcal{W}$ is called low rank if $r_{i}<n_{i}$.

\subsection{Overlapped Trace Norm}

One of the earliest definitions of a tensor norm is the tensor nuclear norm (Liu et al., 2009) or the overlapped trace norm (Tomioka and Suzuki, 2013), which can be represented for a tensor $\mathcal{W} \in \mathbb{R}^{n_{1} \times \cdots \times n_{K}}$ as

$$
\|\mathcal{W}\|_{\text {overlap }}=\sum_{k=1}^{K}\left\|W_{(k)}\right\|_{\text {tr }} .
$$

The overlapped trace norm can be viewed as a direct extension of the matrix trace norm since it unfolds a tensor on each of its mode and computes the sum of trace norms of the unfolded matrices. Regularization with the overlapped trace norm can also be seen as an overlapped group regularization due to the fact that the same tensor is unfolded over different modes and regularized with the trace norm.

One of the popular applications of the overlapped trace norm is tensor completion (Gandy et al., 2011; Liu et al., 2009), where missing entries of a tensor are imputed. Another application is multilinear multitask learning (Romera-Paredes et al., 2013), where multiple vector-based linear learning tasks with a common feature space are arranged as a tensor feature structure and the multiple tasks are solved together with constraints to minimize the multilinear ranks of the tensor feature.

Theoretical analyses on the overlapped norm have been carried out for both tensor completion (Tomioka and Suzuki, 2013) and multilinear multitask learning (Wimalawarne et al., 2014); they have shown that the prediction error of overlapped trace norm regularization is bounded by the average mode- $k$ ranks which can be large if some modes are close to full rank even if there are low-rank modes. Thus, these studies imply that the overlapped trace norm performs well when the multilinear ranks have small variations, and it may result in a poor performance when the multilinear ranks have high variations.

To overcome the weakness of the overlapped trace norm, recent research in tensor norms has led to new norms such as the latent trace norm (Tomioka and Suzuki, 2013) and the scaled latent trace norm (Wimalawarne et al., 2014). 


\subsection{Latent Trace Norm}

Tomioka and Suzuki (2013) proposed the latent trace norm as

$$
\|\mathcal{W}\|_{\text {latent }}=\inf _{\mathcal{W}^{(1)}+\mathcal{W}^{(2)}+\ldots+\mathcal{W}^{(K)}=\mathcal{W}} \sum_{k=1}^{K}\left\|W_{(k)}^{(k)}\right\|_{\text {tr }} .
$$

The latent trace norm takes a mixture of $K$ latent tensors which is equal to the number of modes, and regularizes each of them separately. In contrast to the overlapped trace norm, the latent tensor trace norm regularizes different latent tensors for each unfolded mode and this gives the tendency that the latent tensor trace norm picks the latent tensor with the lowest rank.

In general, the latent trace norm results in a mixture of latent tensors and the content of each latent tensor would depend on the rank of its unfolding. In an extreme case, for a tensor with all its modes full except one mode, regularization with the latent tensor trace norm would result in making the latent tensor with the lowest mode become prominent while others become zero.

\subsection{Scaled Latent Trace Norm}

Recently, Wimalawarne et al. (2014) proposed the scaled latent trace norm as an extension of the latent trace norm:

$$
\|\mathcal{W}\|_{\text {scaled }}=\inf _{\mathcal{W}^{(1)}+\mathcal{W}^{(2)}+\ldots+\mathcal{W}^{(K)}=\mathcal{W}} \sum_{k=1}^{K} \frac{1}{\sqrt{n_{k}}}\left\|W_{(k)}^{(k)}\right\|_{\text {tr }} .
$$

Compared to the latent trace norm, the scaled latent trace norm takes the rank relative to the mode dimension. A major drawback of the latent trace norm is its inability to identify the rank of a mode relative to its dimension. If a tensor has a mode where its dimension is smaller than other modes yet its relative rank with respect to its mode dimension is high compared to other modes, the latent trace norm could incorrectly pick the smallest mode.

The scaled latent norm has the ability to overcome this problem by its scaling with the mode dimensions such that it is able to work with the relative ranks of the tensor. In the context of multilinear multitask learning, it has been shown that the scaled latent trace norm works well for tensors with high variations in multilinear ranks and mode dimensions compared to the overlapped trace norm and the latent trace norm (Wimalawarne et al., 2014).

The inductive learning setting mentioned in (1) with the overlapped trace norm has been studied previously in Signoretto et al. (2013). However, theoretical analysis and performance comparison with other tensor norms have not been conducted yet. Similarly to tensor decomposition (Tomioka and Suzuki, 2013) and multilinear multitask learning (Wimalawarne et al., 2014), tensor-based regression and classification may also be improved by regularization methods that can work with high variations in multilinear ranks and mode dimensions.

In the following sections, to make tensor-based learning more practical and to improve the performance, we consider formulation (1) with the overlapped trace norm, the 
latent trace norm, and the scaled latent trace norm, and give computationally efficient optimization algorithms and excess risk bounds.

\section{Optimization}

In this section, we consider the dual formulation for (11) and propose computationally efficient optimization algorithms. Since optimization of (1) with regularization using the overlapped trace norm has already been studied in Signoretto et al. (2013), we do not discuss it again here. Our main focus in this section is optimization of (1) with regularization using the latent trace norm and the scaled latent trace norm.

Let us consider the formulation (11) for a data set $\left(\mathcal{X}_{i}, y_{i}\right) \in \mathbb{R}^{n_{1} \times \cdots \times n_{K}} \times \mathbb{R}, i=1, \ldots, m$ with latent and scaled latent trace norm regularization as follows:

$$
P(\mathcal{W}, b)=\min _{\mathcal{W}^{(1)}+\ldots+\mathcal{W}^{(K)}=\mathcal{W}, b} \sum_{i=1}^{m} l\left(\mathcal{X}_{i}, y_{i}, \mathcal{W}, b\right)+\sum_{k=1}^{K} \lambda_{k}\left\|W_{(k)}^{(k)}\right\|_{\mathrm{tr}},
$$

where, for $k=1, \ldots, K$ and for any given regularization parameter $\lambda, \lambda_{k}=\lambda$ in the case of the latent trace norm and $\lambda_{k}=\frac{\lambda}{\sqrt{n_{k}}}$ in the case of the scaled latent trace norm, respectively. $W_{(k)}^{(k)}$ is the unfolding of $\mathcal{W}^{(k)}$ on its $k$ th mode. It is worth noticing that the application of the latent and scaled latent trace norms requires optimizing over $K$ latent tensors which contain $K N$ variables in total. For large $K$ and $N$, solving the primal problem (6) can be computationally expensive especially in non-linear problems such as logistic regression, since they require computationally expensive optimization methods such as gradient descent or the Newton method. If the number of training samples $m$ is $m \ll K N$, solving the dual problem of (6) could be computationally more efficient. For this reason, we focus on optimization in the dual below.

The dual formulation of (6) can be written as follows (its detailed derivation is given in Appendix A):

$$
\begin{gathered}
\min _{\boldsymbol{\alpha}, \mathcal{V}^{(1)}, \cdots, \mathcal{V}^{(K)}} D(-\boldsymbol{\alpha})+\sum_{k=1}^{K} \delta_{\lambda_{k}}\left(V_{(k)}^{(k)}\right) \\
\text { ssutject to } \mathcal{V}^{(k)}=\sum_{i=1}^{m} \alpha_{i} \mathcal{X}_{i} \quad(k=1, \ldots, K,) \\
\sum_{i=1}^{m} \alpha_{i}=0,
\end{gathered}
$$

where $\boldsymbol{\alpha}=\left(\alpha_{1}, \ldots, \alpha_{m}\right)^{\top} \in \mathbb{R}^{m}$ are dual variables corresponding to the training data set $\left(\mathcal{X}_{i}, y_{i}\right), i=1, \ldots, m, D(-\boldsymbol{\alpha})$ is the conjugate loss function defined as

$$
D(-\boldsymbol{\alpha})=\sum_{i=1}^{m} \frac{1}{2} \alpha_{i}^{2}-\alpha_{i} y_{i}
$$

in the case of regression with the squared loss (Tomioka et al., 2011c), and

$$
D(-\boldsymbol{\alpha})=\sum_{i=1}^{m} y_{i} \alpha_{i} \log \left(y_{i} \alpha_{i}\right)+\left(1-y_{i} \alpha_{i}\right) \log \left(1-y_{i} \alpha_{i}\right)
$$


with constraint $0 \leq y_{i} \alpha_{i} \leq 1$ in the case of classification with the logistic loss (Tomioka et al., 2011c). $\delta_{\lambda_{k}}$ is the indicator function defined as $\delta_{\lambda_{k}}(V)=0$ if $\|V\|_{\text {op }} \leq \lambda_{k}$ and $\delta_{\lambda_{k}}(V)=\infty$ otherwise. The constraint $\quad \sum_{i=1}^{m} \alpha_{i}=0$ is due to the bias term $b$. Here, the auxiliary variables $\mathcal{V}^{(1)}, \ldots, \mathcal{V}^{(N)}$ are introduced to remove the coupling between the indicator functions in the objective function (see Appendix A for details).

The alternating direction method of multipliers (ADMM) (Gabay and Mercier, 1976; Boyd et al., 2011) has been previously used to solve primal problems of tensor decomposition (Tomioka et al., 2011b) and multilinear multi-task learning (Romera-Paredes et al., 2013) with the overlapped trace norm regularization. Optimization in the dual for tensor decomposition problems with the latent and scaled latent trace norm regularization has been solved using ADMM in Tomioka et al. (2011b). Here, we also adopt ADMM to solve (7), and describe the formulation and the optimization steps in detail.

With introduction of dual variables $\mathcal{W}^{(k)} \in \mathbb{R}^{n_{1} \times \cdots \times n_{K}}, k=1, \ldots, K$ (corresponding to the primal variables of (6) $), b \in \mathbb{R}$, and parameter $\beta>0$, the augmented Lagrangian function for (7) is defined as follows:

$$
\begin{aligned}
L\left(\boldsymbol{\alpha},\left\{\mathcal{V}^{(k)}\right\}_{k=1}^{K},\left\{\mathcal{W}^{(k)}\right\}_{k=1}^{K}, b\right) & \\
=D(-\boldsymbol{\alpha})+\sum_{k=1}^{K} & \left(\delta_{\lambda_{k}}\left(V_{(k)}^{(k)}\right)+\left\langle W_{(k)}^{(k)}, \sum_{i=1}^{m} \alpha_{i} X_{i(k)}-V_{(k)}^{(k)}\right\rangle\right. \\
& \left.+\frac{\beta}{2}\left\|\sum_{i=1}^{m} \alpha_{i} X_{i(k)}-V_{(k)}^{(k)}\right\|_{\mathrm{F}}^{2}\right)+b \sum_{i=1}^{m} \alpha_{i}+\frac{\beta}{2}\left\|\sum_{i=1}^{m} \alpha_{i}\right\|_{\mathrm{F}}^{2} .
\end{aligned}
$$

This ADMM formulation is solved for variables $\boldsymbol{\alpha}, \mathcal{V}^{(1)}, \ldots, \mathcal{V}^{(k)}, \mathcal{W}^{(1)}, \ldots, \mathcal{W}^{(k)}$, and $b$ by considering sub-problems for each variable. Below, we give the solution for each variable at iterative step $t+1$.

The first sub-problem to solve is for $\boldsymbol{\alpha}$ at step $t+1$ :

$$
\boldsymbol{\alpha}^{t+1}=\underset{\boldsymbol{\alpha}}{\operatorname{argmax}} L\left(\boldsymbol{\alpha},\left\{\mathcal{V}^{(k) t}\right\}_{k=1}^{K},\left\{\mathcal{W}^{(k) t}\right\}_{k=1}^{K}, b^{t}\right),
$$

where $\left\{\mathcal{V}^{(k) t}\right\}_{k=1}^{K},\left\{\mathcal{W}^{(k) t}\right\}_{k=1}^{K}$, and $b^{t}$ are the solutions obtained at step $t$.

Depending on the conjugate loss $D(-\boldsymbol{\alpha})$, the solution for $\boldsymbol{\alpha}$ differs. In the case of regression with the squared loss (2), the augmented Lagrangian can be minimized with respect to $\boldsymbol{\alpha}$ by solving the following linear equation:

$$
\left(K \beta \bar{X} \bar{X}^{\top}+I+\beta \mathbf{1}_{m} \mathbf{1}_{m}^{\top}\right) \boldsymbol{\alpha}^{t+1}=\left(\mathbf{y}-\bar{X} \operatorname{vec}\left(\overline{\mathcal{W}}^{t}\right)+\beta \bar{X} \operatorname{vec}\left(\overline{\mathcal{V}}^{t}\right)-\mathbf{1}_{m} b^{t}\right),
$$

where $\bar{X}=\left[\operatorname{vec}\left(\mathcal{X}_{1}\right)^{\top} ; \cdots ; \operatorname{vec}\left(\mathcal{X}_{m}\right)^{\top}\right] \in \mathbb{R}^{m \times N}, \overline{\mathcal{V}}^{t}=\sum_{k=1}^{K} \mathcal{V}^{(k) t}, \overline{\mathcal{W}}^{t}=\sum_{k=1}^{K} \mathcal{W}^{(k) t}$, $\mathbf{y}=\left(y_{1}, \ldots, y_{m}\right)^{\top}$, and $\mathbf{1}_{m}$ is the $m$-dimensional vector of all ones. Note that, in the above system of equations, coefficient matrix multiplied with $\boldsymbol{\alpha}$ does not change during optimization. Thus, it can be efficiently solved at each iteration by precomputing the Cholesky factorization of the matrix.

For classification with the logistic loss (3), the Newton method is used to find the solution for $\boldsymbol{\alpha}^{t+1}$, which requires the gradient and the Hessian of 


$$
\begin{aligned}
L\left(\boldsymbol{\alpha},\left\{\mathcal{V}^{(k)}\right\}_{k=1}^{K},\left\{\mathcal{W}^{(k)}\right\}_{k=1}^{K}, b\right): & \begin{aligned}
\frac{\partial L\left(\boldsymbol{\alpha},\left\{\mathcal{V}^{(k)}\right\}_{k=1}^{K},\left\{\mathcal{W}^{(k)}\right\}_{k=1}^{K}, b\right)}{\partial \alpha_{i}}= & y_{i} \log \left(\frac{y_{i} \alpha_{i}}{1-y_{i} \alpha_{i}}\right)+\sum_{k=1}^{K}\left\langle\mathcal{W}^{(k) t}, \mathcal{X}_{i}\right\rangle \\
& +\beta \sum_{k=1}^{K}\left\langle\mathcal{X}_{i}, \sum_{i=1}^{m} \mathcal{X}_{i} \alpha_{i}^{t+1}-\mathcal{V}^{(k) t}\right\rangle+b+\beta \sum_{i=1}^{m} \alpha_{i},
\end{aligned} \\
\frac{\partial^{2} L\left(\boldsymbol{\alpha},\left\{\mathcal{V}^{(k)}\right\}_{k=1}^{K},\left\{\mathcal{W}^{(k)}\right\}_{k=1}^{K}, b\right)}{\partial \alpha_{i} \partial \alpha_{j}}= & \begin{cases}\frac{1}{y_{i} \alpha_{i}\left(1-y_{i} \alpha_{i}\right)}+K \beta\left\langle\mathcal{X}_{i}, \mathcal{X}_{i}\right\rangle+\beta & (i=j), \\
K \beta\left\langle\mathcal{X}_{i}, \mathcal{X}_{j}\right\rangle+\beta & (i \neq j) .\end{cases}
\end{aligned}
$$

Next, we update $\mathcal{V}^{(k)}$ at step $t+1$ by solving the following sub-problem:

$$
\begin{aligned}
\mathcal{V}^{(k)^{t+1}} & =\underset{\mathcal{V}(k)}{\operatorname{argmax}} L\left(\boldsymbol{\alpha}^{t+1}, \mathcal{V}^{(k)},\left\{\mathcal{V}^{(j) t}\right\}_{j \neq k}^{K},\left\{\mathcal{W}^{(k) t}\right\}_{k=1}^{K}, b^{t}\right) \\
& =\operatorname{proj}_{\lambda_{k}}\left(\frac{W_{(k)}^{(k) t}}{\beta}+\sum_{i=1}^{m} \alpha_{i}^{t+1} X_{i(k)}\right),
\end{aligned}
$$

where $\operatorname{proj}_{\lambda}(W)=U \min (S, \lambda) V^{T}$ and $W=U S V^{\top}$.

Finally, we update the dual variables $\mathcal{W}^{(k)}$ and $b$ at step $t+1$ as

$$
\begin{aligned}
W_{(k)}^{(k) t+1} & =W_{(k)}^{(k) t}+\beta\left(\sum_{i=1}^{m} \alpha_{i}^{t+1} X_{i(k)}-V_{(k)}^{(k) t+1}\right), \\
b^{t+1} & =b^{t}+\beta \sum_{i=1}^{m} \alpha_{i}^{t+1} .
\end{aligned}
$$

Note that step (8) and step (9) can be combined as

$$
W_{(k)}^{(k) t+1}=\operatorname{prox}_{\beta \lambda_{k}}\left(W_{(k)}^{(k) t}+\beta \sum_{i=1}^{m} \alpha_{i}^{t+1} X_{i(k)}\right),
$$

where $\operatorname{prox}_{\lambda}(W)=U \max (S-\lambda, 0) V^{T}$ and $W=U S V^{\top}$. This allows us to avoid computing singular values and the associated singular vectors that are smaller than the threshold $\lambda_{k}$ in (8) .

\section{Optimality Condition}

As a stopping condition, we use the relative duality gap (Tomioka et al., 2011a), which can be expressed as

$$
\frac{P\left(\mathcal{W}^{t}, b^{t}\right)-D\left(-\hat{\boldsymbol{\alpha}}^{t}\right)}{P\left(\mathcal{W}^{t}, b^{t}\right)} \leq \epsilon
$$

where $P\left(\mathcal{W}^{t}, b^{t}\right)$ is the primal solution at step $t$ of (6) and $\epsilon$ is a predefined tolerance value. $D\left(-\hat{\boldsymbol{\alpha}}^{t}\right)$ is the dual solution at step $t$ of (7) with $\hat{\boldsymbol{\alpha}}$ obtained by multiplying $\boldsymbol{\alpha}$ with $\min \left(1, \frac{\left\|V(\boldsymbol{\alpha})_{(k)}\right\|_{\mathrm{op}}}{\lambda_{1}}, \ldots, \frac{\left\|V(\boldsymbol{\alpha})_{(k)}\right\|_{\mathrm{op}}}{\lambda_{K}}\right)$, where $\mathcal{V}(\boldsymbol{\alpha})=\sum_{i=1}^{m} X_{i} \alpha_{i}$ and $\|V\|_{\text {op }}$ is the largest singular value of $V$. 


\section{Theoretical Risk Analysis}

In this section, we theoretically analyze the excess risk for regularization with the overlapped trace norm, the latent trace norm, and the scaled latent trace norm.

We consider a loss function $l$ which is Lipshitz continuous with constant $\Lambda$. Note that this condition is true for both the squared loss and logistic loss functions. Let the training data set be given as $\left(\mathcal{X}_{i}, y_{i}\right) \in \mathbb{R}^{n_{1} \times \cdots \times n_{K}} \times \mathrm{Y}, i=1, \ldots, m$, where $\mathrm{Y} \in \mathbb{R}$ for regression and $\mathrm{Y} \in\{-1,1\}$ for classification. In our theoretical analysis, we assume that elements of $\mathcal{X}_{i}$ independently follow the standard Gaussian distribution.

As the standard formulation (Maurer and Pontil, 2013), the empirical risk without the bias term is defined as

$$
\hat{R}(\mathcal{W})=\frac{1}{m} \sum_{i=1}^{m} l\left(\left\langle\mathcal{W}, \mathcal{X}_{i}\right\rangle, y_{i}\right),
$$

and the expected risk is defined as

$$
R(\mathcal{W})=\mathbb{E}_{(\mathcal{X}, y) \sim \mu} l(\langle\mathcal{W}, \mathcal{X}\rangle, y),
$$

where $\mu$ is the probability distribution from which $\left(\mathcal{X}_{i}, y_{i}\right)$ are sampled.

The optimal $\mathcal{W}^{0}$ that minimizes the expected risk is given as

$$
\mathcal{W}^{0}=\arg \min _{\mathcal{W}} R(\mathcal{W}) \quad \text { subject to }\|\mathcal{W}\|_{\star} \leq B_{0}
$$

where $\|\cdot\|_{\star}$ is either the overlapped trace norm, the latent trace norm, or the scaled latent trace norm. The optimal $\hat{\mathcal{W}}$ that minimizes the empirical risk is denoted as

$$
\hat{\mathcal{W}}=\arg \min _{\mathcal{W}} \hat{R}(\mathcal{W}) \quad \text { subject to }\|\mathcal{W}\|_{\star} \leq B_{0}
$$

The next lemma provides an upper bound of the excess risk for tensor-based learning problems (see Appendix B for its proof), where $\|\mathcal{W}\|_{\star^{*}}$ is the dual norm of $\|\mathcal{W}\|_{\star}$ for $\star=\{$ overlap, latent, scaled $\}$ :

Lemma 1. For a given $\Lambda$-Lipchitz continuous loss function l and for any $\mathcal{W} \in \mathbb{R}^{n_{1} \times \cdots \times n_{K}}$ such that $\|\mathcal{W}\|_{\star} \leq B_{0}$ for problems (11) -(12) , the excess risk for a given training data set $\left(\mathcal{X}_{i}, y_{i}\right) \in \mathbb{R}^{n_{1} \times \cdots \times n_{K}} \times \mathbb{R}, i=1, \ldots, m$ is bounded with probability at least $1-\delta$ as

$$
R(\hat{\mathcal{W}})-R\left(\mathcal{W}^{0}\right) \leq \frac{2}{m} \Lambda B_{0} \mathbb{E}\|\mathcal{M}\|_{\star^{*}}+\sqrt{\frac{\log \left(\frac{2}{\delta}\right)}{2 m}}
$$

where $\mathcal{M}=\sum_{i=1}^{m} \sigma_{i} \mathcal{X}_{i}$ and $\sigma_{i} \in\{-1,1\}$ are Rademacher random variables.

The next theorem gives an excess risk bound for overlapped trace norm regularization (its proof is also included in Appendix B), which is based on the inequality $\|\mathcal{W}\|_{\text {overlap }} \leq$ $\sum_{k=1}^{K} \sqrt{r_{k}}\|\mathcal{W}\|_{\mathrm{F}}$ given in Tomioka and Suzuki (2013): 
Theorem 1. With probability at least $1-\delta$, the excess risk of learning using the overlapped trace norm regularization for any $\mathcal{W}^{0}$ with $\left\|\mathcal{W}^{0}\right\|_{\mathrm{F}} \leq B$, multilinear ranks $\left(r_{1}, \ldots, r_{K}\right)$, and estimator $\hat{\mathcal{W}}$ with $B_{0} \leq B \sum_{k=1}^{K} \sqrt{r_{k}}$ is bounded as

$$
R(\hat{W})-R\left(W^{0}\right) \leq c_{1} \Lambda \frac{B}{\sqrt{m}}\left(\sum_{k=1}^{K} \sqrt{r_{k}}\right) \min _{k}\left(\sqrt{n_{k}}+\sqrt{n_{\backslash k}}\right)+c_{2} \sqrt{\frac{\log \left(\frac{2}{\delta}\right)}{2 m}}
$$

where $n_{\backslash k}=\prod_{j \neq k}^{K} n_{j}$ and $c_{1}$ and $c_{2}$ are constants.

In the next theorem, we give an excess risk bound for the latent trace norm (its proof is also included in Appendix B), which uses the inequality $\|\mathcal{W}\|_{\text {latent }} \leq \sqrt{\min _{k} r_{k}}\|\mathcal{W}\|_{\mathrm{F}}$ given in Tomioka and Suzuki (2013):

Theorem 2. With probability at least $1-\delta$, the excess risk of learning using the latent norm regularization for any $\mathcal{W}^{0}$ with $\left\|\mathcal{W}^{0}\right\|_{\mathrm{F}} \leq B$, multilinear ranks $\left(r_{1}, \ldots, r_{K}\right)$, and estimator $\hat{\mathcal{W}}$ with $B_{0} \leq B \sqrt{\min _{k} r_{k}}$ is bounded as

$$
R(\hat{W})-R\left(W^{0}\right) \leq c_{1} \Lambda B \sqrt{\frac{\min _{k} r_{k}}{m}}\left(\max _{k}\left(\sqrt{n_{k}}+\sqrt{n_{\backslash k}}\right)+C \sqrt{2 \log (K)}\right)+c_{2} \sqrt{\frac{\log \left(\frac{2}{\delta}\right)}{2 m}}
$$

where $n_{\backslash k}=\prod_{j \neq k}^{K} n_{j}$ and $c_{1}, c_{2}$, and $C$ are constants.

The above theorem shows that the excess risk for the latent trace norm (15) is bounded by the minimum multilinear rank. If $n_{1}=\cdots=n_{K}$, the latent trace norm is always better then the overlapped trace norm in terms of the excess risk bounds because $\sqrt{\min _{k} r_{k}}<$ $\sum_{k=1}^{K} \sqrt{r_{k}}$. If the dimensions $n_{1}, \ldots, n_{K}$ are not the same, the overlapped trace norm could be better.

Finally, we bound the excess risk for the scaled latent trace norm based on the inequality $\|\mathcal{W}\|_{\text {scaled }} \leq \sqrt{\min _{k}\left(\frac{r_{k}}{n_{k}}\right)}\|\mathcal{W}\|_{\text {F }}$ given in Wimalawarne et al. (2014):

Theorem 3. With probability at least $1-\delta$, the excess risk of learning using the scaled latent trace norm regularization for any $\mathcal{W}^{0}$ with $\left\|\mathcal{W}^{0}\right\|_{\mathrm{F}} \leq B$, multilinear ranks $\left(r_{1}, \ldots, r_{K}\right)$, and estimator $\hat{\mathcal{W}}$ with $B_{0} \leq B \sqrt{\min _{k}\left(\frac{r_{k}}{n_{k}}\right)}$ is bounded as

$$
R(\hat{W})-R\left(W^{0}\right) \leq c_{1} \Lambda B \sqrt{\frac{1}{m} \min _{k}\left(\frac{r_{k}}{n_{k}}\right)}\left(\max _{k}\left(n_{k}+\sqrt{N}\right)+C \sqrt{2 \log (K)}\right)+c_{2} \sqrt{\frac{\log \left(\frac{2}{\delta}\right)}{2 m}},
$$

where $c_{1}, c_{2}$, and $C$ are constants.

Note that when $n_{1}=\cdots=n_{K}=n$ and the multilinear ranks $r_{1}, \ldots, r_{K}$ are different, the bounds in Theorem 2 and Theorem 3 are the same.

Theorem 3 shows that the excess risk for regularization with the scaled latent trace norm is bounded with the minimum of multilinear ranks relative to their mode dimensions. Similarly to the latent trace norm, the scaled latent trace norm would also perform 
better than the overlapped norm when the multilinear ranks have large variations. If we consider a "flat" tensor, the modes with small dimensions may have ranks comparable to their dimensions. Although these modes have the lowest mode- $k$ rank, they do not impose a low-rank structure. In such cases, our theory predicts that the scaled latent trace norm performs better because it is sensitive to the mode- $k$ rank relative to its dimension.

As a variation, we can also consider a mode-wise "scaled" version of the overlapped trace norm defined as $\|\mathcal{W}\|_{\text {soverlap }}:=\sum_{k=1}^{K} \frac{1}{\sqrt{n_{k}}}\left\|W_{(k)}\right\|_{\text {tr. }}$. It can be easily seen that $\|\mathcal{W}\|_{\text {soverlap }} \leq \sum_{k=1}^{K} \sqrt{\frac{r_{k}}{n_{k}}}\|\mathcal{W}\|_{\mathrm{F}}$ holds and with the same conditions as in Theorem 1 , we can upper-bound the excess risk for the scaled overlapped trace norm regularization as

$$
R(\hat{W})-R\left(W^{0}\right) \leq c_{1} \Lambda \frac{B}{\sqrt{m}}\left(\sum_{k=1}^{K} \sqrt{\frac{r_{k}}{n_{k}}}\right) \min _{k}\left(n_{k}+\sqrt{N}\right)+c_{2} \sqrt{\frac{\log \left(\frac{2}{\delta}\right)}{2 m}} .
$$

Note that when all modes have the same dimensions, (17) coincides with (14). Compared with bound (16), the scaled latent norm would perform better than the scaled overlapped norm regularization since $\min _{k} \sqrt{\frac{r_{k}}{n_{k}}}<\sum_{k=1}^{K} \sqrt{\frac{r_{k}}{n_{k}}}$.

\section{$5 \quad$ Experiments}

We conducted several experiments using simulated and real-world data to evaluate the performance of tensor-based regression and classification methods with regularizations using different tensor norms. We discuss simulations for tensor-based regression in Section 5.1, experiments with real-world data for tensor classification in Section 5.2. For all experiments, we use a MATLAB ${ }^{\circledR}$ environment on a $2.10 \mathrm{GHz}(2 \times 8$ cores $)$ Intel Xeon E5-2450 server machine with 128 GB memory.

\subsection{Tensor Regression with Artificial Data}

We report the results of artificial data experiments on tensor-based regression.

We generated three different 3-mode tensors as weight tensors $\mathcal{W}$ with different multilinear ranks and mode dimensions. We created two homogenous tensors with equal mode dimensions of $n_{1}=n_{2}=n_{3}=10$ with different multilinear ranks $\left(r_{1}, r_{2}, r_{3}\right)=(3,3,3)$ and $\left(r_{1}, r_{2}, r_{3}\right)=(3,5,8)$. The third weight tensor is an inhomogenous case with mode dimensions of $n_{1}=4, n_{2}=n_{3}=10$ and multilinear ranks $\left(r_{1}, r_{2}, r_{3}\right)=(3,4,8)$. To generate these weight tensors, we use the Tucker decomposition (Kolda and Bader, 2009) of a tensor as $\mathcal{W}=\mathcal{C} \times{ }_{k=1}^{3} U^{(k)}$, where $\mathcal{C} \in \mathbb{R}^{r_{1} \times r_{2} \times r_{3}}$ is the core tensor and $U^{(k)} \in \mathbb{R}^{r_{k} \times n_{k}}$ are component matrices. We sample elements of the core tensor $\mathcal{C}$ from a standard Gaussian distribution, choose component matrices $U^{(k)} \in \mathbb{R}^{r_{k} \times n_{k}}$ to be orthogonal matrices, and generate $\mathcal{W}$ by mode-wise multiplication of the core tensor and component matrices.

To create training samples $\left\{\mathcal{X}_{i}, y_{i}\right\}_{i=1}^{n}$, we first create the random tensors $\mathcal{X}_{i}$ generated with each element independently sampled from the standard Gaussian distribution and obtain $y_{i}=\left\langle\mathcal{W}, \mathcal{X}_{i}\right\rangle+\nu_{i}$, where $\nu_{i}$ is noise drawn from the Gaussian distribution with mean zero and variance 0.1 . In our experiments we use cross validation to select the regularization parameter from range $0.01-100$ at intervals of 0.1 . For the purpose of 
comparison, we have also simulated matrix regularized regressions for each mode unfolding. Also, we experimented with cross validation among matrix regularization on each unfolded matrix to understand whether it can find the correct mode for regularization. As the baseline vector-based learning method, we use ridge regression (i.e., $l_{2}$-regularized least-squares).

Figure 1 shows the performance of homogenous tensors with equal mode dimensions $n_{1}=n_{2}=n_{3}=10$ and equal multilinear ranks $\left(r_{1}, r_{2}, r_{3}\right)=(3,3,3)$. We see that the overlapped norm performs the best, while both latent norms perform equally (since mode dimensions are equal) but inferior to the overlapped norm. Also, the regression results from all matrix regularizations with individual modes perform better than the latent and the scaled latent norm regularized regression models. Due to the equal multilinear ranks and equal mode dimensions, it results in equal performance with cross validation among each mode-wise unfolded matrix regularization.

Figure 2 shows the performances of homogenous tensors with equal mode dimensions $n_{1}=n_{2}=n_{3}=10$ and unequal multilinear ranks $\left(r_{1}, r_{2}, r_{3}\right)=(3,5,8)$. In this case, both the latent and the scaled latent norms also perform equally since tensor dimensions are the same. The mode- 1 regularized regression models give the best performance since it has the lowest rank and regularization with the latent and scaled latent norms gives the next best performance. The mode-wise cross validation correctly coincides with the mode-1 regularization. The overlapped norm performs poorly compared to the latent and the scaled latent trace norms.

Figure 3 shows the performance of inhomogenous tensors with mode dimensions $n_{1}=$ 4, $n_{2}=n_{3}=10$ and multilinear ranks $\left(r_{1}, r_{2}, r_{3}\right)=(3,4,8)$. In this case, we can see that the scaled latent trace norm outperforms all other tensor norms. The latent trace norm performs poorly since it fails to find the mode with the lowest rank. This well agrees with our theoretical analysis: as shown in (15), the excess risk of the latent trace norm is bounded with the minimum of multilinear ranks, which is on the first mode in the current setup and it is high ranked. The scaled latent trace norm is able to find the mode with the lowest rank since it takes the relative rank with respect to the mode dimension as in (16). If we look at the individual mode regularizations, we see that the best performance is given with the second mode, which has the lowest rank with respect to the mode dimension, and the worst performance is given with the first mode, which is high ranked compared to other modes. Here, the mode-wise cross validation is again as good as mode-2 regularization.

It is also worth noticing in all above experiments that ridge regression performed worse than all the tensor regularized learning models. This highlights the necessity of employing low-rank inducing norms for learning with tensor data without vectorization to get the best performance.

Figure 4 shows the computation time for the toy regression experiment with inhomogenous tensors with mode dimensions $n_{1}=4, n_{2}=n_{3}=10$ and multilinear ranks $\left(r_{1}, r_{2}, r_{3}\right)=(3,4,8)$ (computation time for other setups showed similar tendency and thus we omit the results). For each data set, we measured the computation time of training regression models, cross validation for model selection, and predicting output values for test data. We can see that methods based on tensor norms and matrix norms are computationally much more expensive compared to ridge regression. However, as we saw above, they achieves higher accuracy than ridge regression. It is worth noticing that 


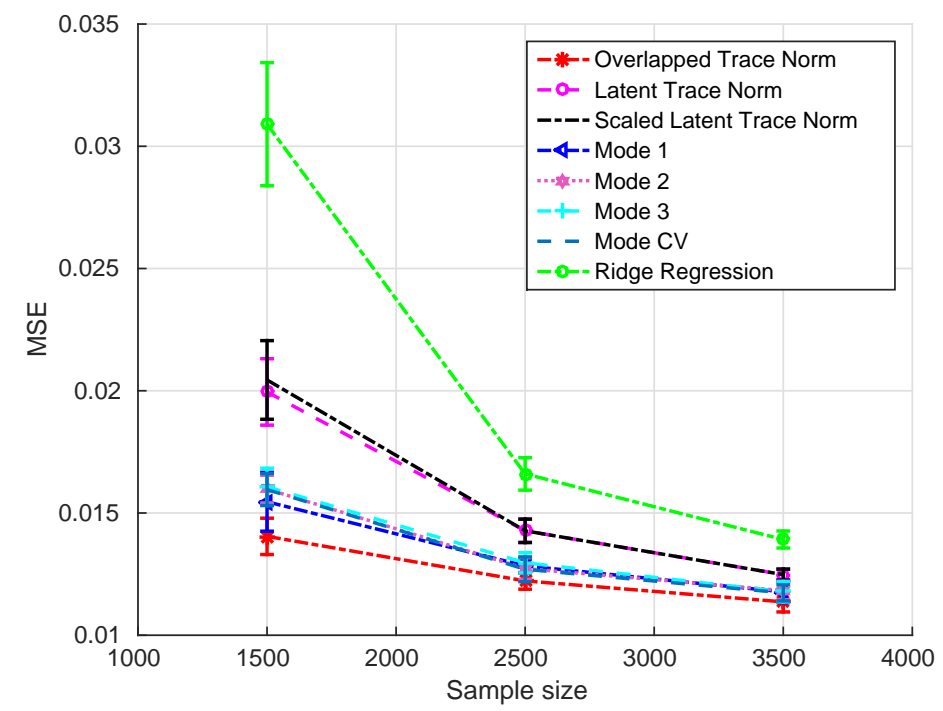

Figure 1: Simulation results of tensor regression based on homogenous weight tensor of equal mode dimensions $n_{1}=n_{2}=n_{3}=10$ and equal multilinear $\operatorname{ranks}\left(r_{1}, r_{2}, r_{3}\right)=$ $(3,3,3)$

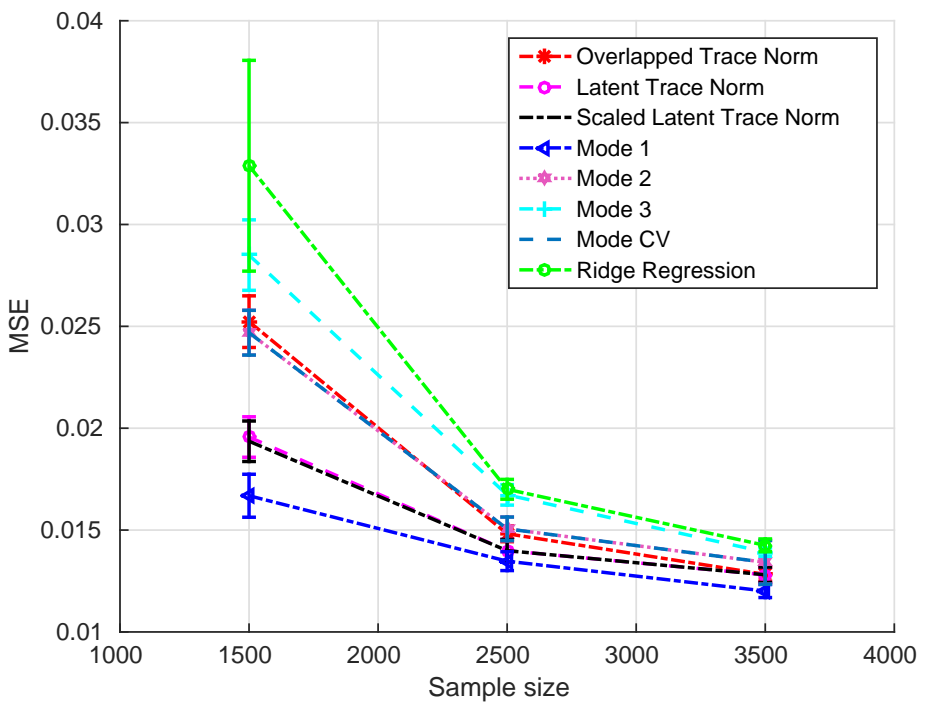

Figure 2: Simulation results of tensor regression based on homogenous weight tensor of equal modes sizes $n_{1}=n_{2}=n_{3}=10$ and unequal multilinear rank $\left(r_{1}, r_{2}, r_{3}\right)=(3,5,8)$ 


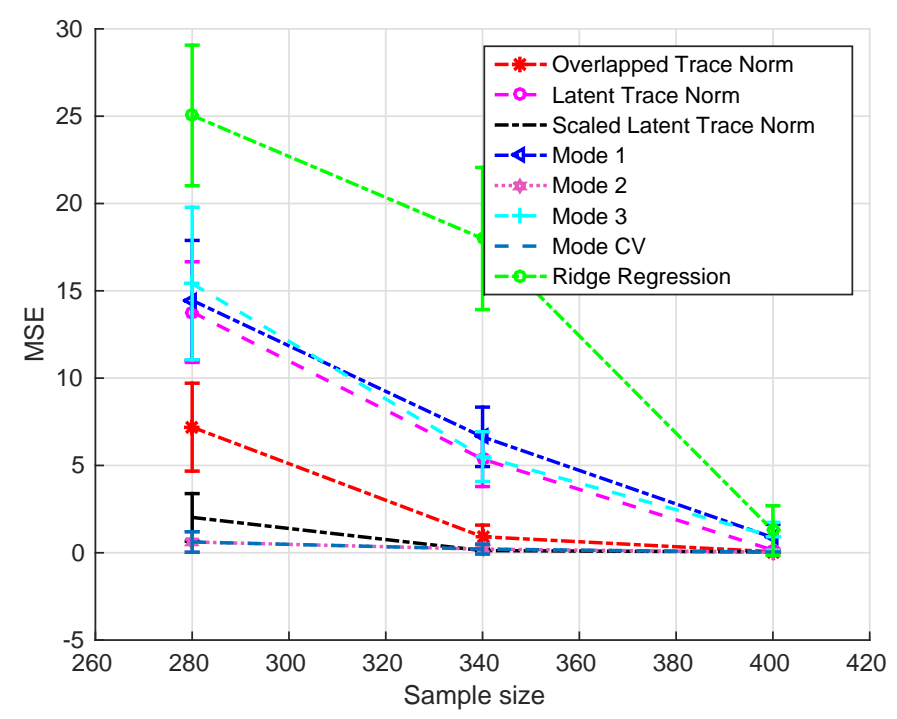

Figure 3: Simulation results of tensor regression based on inhomogenous weight tensor of equal modes sizes $n_{1}=4, n_{2}=n_{3}=10$ and multilinear $\operatorname{rank}\left(r_{1}, r_{2}, r_{3}\right)=(3,4,8)$

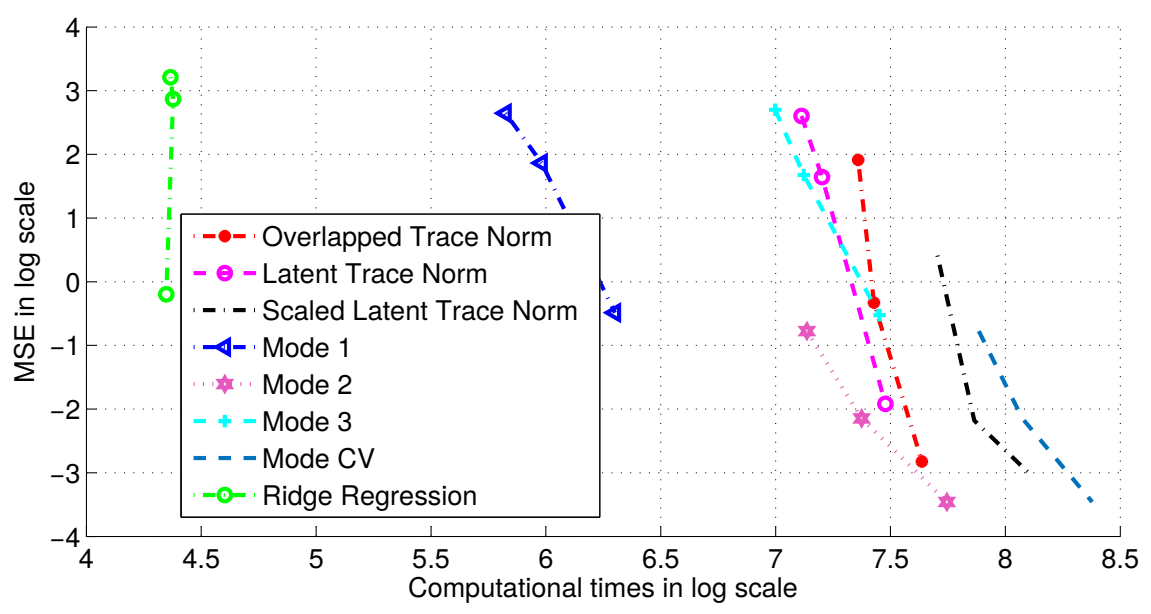

Figure 4: Computation times in seconds for toy experiment with inhomogenous tensors with mode dimensions $n_{1}=4, n_{2}=n_{3}=10$ and multilinear rank $\left(r_{1}, r_{2}, r_{3}\right)=(3,4,8)$ 


\section{Flat/Leftward}

Spread/Leftward

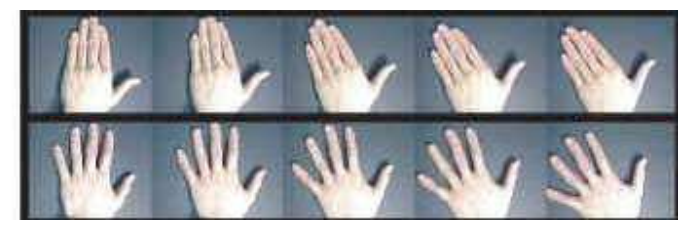

Figure 5: Samples of hand motion sequences of left/flat and left/spread

mode-wise cross validation is computationally more expensive compared to the scaled latent trace norm and other tensor norms. This computational advantage and comparable performance with respect to the best mode-wise regularization makes the scaled latent trace norm a useful regularization method for tensor-based regression especially for tensors with high variations in its multilinear ranks.

\subsection{Tensor Classification for Hand Gesture Recognition}

Next, we report the results of experiments on tensor classification with the Cambridge hand gesture data set (Kim et al., 2007).

The Cambridge hand gesture data set contains image sequences from 9 gesture classes. These gesture classes include 3 primitive hand shapes of flats, spread, and V-shape, and 3 different hand motions of rightward, leftward, and contrast. Each class has 100 image sequences with different illumination conditions and arbitrary motions of two people. Previously, the tensor canonical correlation (Kim et al., 2007) has been used to classify these hand gestures.

To apply tensor classification, first we build action sequences as tensor data by sampling $S$ images with equal time intervals from each sequence. This makes each sequence a tensor of $20 \times 20 \times S$, where the first two modes are down-sampled images as in (Kim et al., 2007) and $S$ is the number of sampled images. In our experiments, we set $S$ at 5 or 10 . We consider binary classification and we have chosen visually similar sequences of left/flat and left/spread (Figure 5), which we found to be difficult to classify. We apply standardization of data by mean removal and variance normalization. We randomly sample data into a training set of 120 data elements, use a validation set of 40 data elements to select the optimal regularization parameter, and finally use a test set of 40 elements to evaluate the learned classifier. In addition to the tensor regularized learning models, we also trained classifiers with matrix regularization with unfolding on each mode separately. As a baseline vector-based learning method, we have used the $l_{2}$-regularized logistic regression. We also trained mode-wise cross validation with individual mode regularization (Mode-wise CV). We repeated the learning procedure for 10 sample sets for each classifier and the results are shown in Table 1.

In both experiments for $S=5$ and 10, we see that tensor norm regularized classification performs better than the vectorized learning method. With tensor structure of $(20,20,5)$, we can see that the scaled latent norm gives the best performance and the latent trace norm, mode-1, mode-3, and mode-wise cross validation gives are comparable. We observed that, with the tensor structure of $(20,20,5)$, the resulted weight tensor after learning its third mode becomes full rank. The scaled latent trace norm performed the best since it could identify the mode with the minimum rank relative to its mode 
Table 1: Classification error of experiments with the hand gesture data set. The boldfaced figures indicate comparable accuracies among classifiers after a t-test with significance of 0.05 .

\begin{tabular}{|c|c|c|}
\hline & \multicolumn{2}{|c|}{ Tensor Dimensions } \\
\hline Norm & $(20,20,5)$ & $(20,20,10)$ \\
\hline Overlapped Trace Norm & $0.1425(0.0512)$ & $0.0722(0.0363)$ \\
\hline Latent Trace Norm & $0.1175(0.0487)$ & $0.0806(0.0512)$ \\
\hline Scaled Latent Trace Norm & $0.0975(0.0478)$ & $0.0944(0.0512)$ \\
\hline Mode-1 & $0.1050(0.0422)$ & $0.0950(0.0438)$ \\
\hline Mode-2 & $0.1400(0.0709)$ & $0.0900(0.0459)$ \\
\hline Mode-3 & $0.1200(0.0405)$ & $0.1100(0.0592)$ \\
\hline Mode-wise CV & $0.1050(0.0542)$ & $0.0950(0.044)$ \\
\hline Logistic regression $\left(l_{2}\right)$ & $0.1975(0.0640)$ & $0.1925(0.0782)$ \\
\hline
\end{tabular}

dimension, which was the first mode in the current setup. The overlapped trace norm performs poorly due to large variations in the multilinear ranks and tensor dimensions.

With the tensor structure $(20,20,10)$, the overlapped trace norm gives the best performance. In this case, we found that the multilinear ranks are close to each other, which made the overlapped norm to give better performance. The scaled latent trace norm, latent trace norm, mode-1, mode-2, and mode-wise cross validation gave comparable performance with the overlapped trace norm.

\subsection{Tensor Classification for Brain Computer Interface}

As our second tensor classification, we experimented with a motor-imagery EEG classification problem in the context of brain computer interface (BCI). The objective of the experiments was to classify movements imagined by person using the EEG signals captured in that instance. For our experiments, we used the data from the BCI competition IVa (Dornhege et al., 2004) . Previous research by Tomioka and Aihara (2007) has considered "channel $\times$ channel" as a matrix of the EEG signal and classified it using logistic regression with low-rank matrix regularization. Our objective is to model EEG data as tensors to incorporate more information and learn to classify using tensor regularization methods. The BCI competition IVa data set consists of BCI experiments of five people. Though BCI experiments have used 256 channels, we only use signals from 49 channels following Tomioka and Aihara (2007) and pre-process each signal from each channel with $Z$ different band-pass filters (Butterworth filters). Let $S_{i} \in \mathbb{R}^{C \times T}$, where $C$ denotes the number of channels and $T$ denotes the time, be the matrix obtained by processing with the $i^{\text {th }}$ filter. As in Tomioka and Aihara (2007), each $S_{i}$ is further processed to make centering and scaling as $\hat{S}_{i}=\frac{1}{\sqrt{T-1}} S_{i}\left(I_{T}-11^{\top}\right)$. Then we obtain $X_{i}=\hat{S}_{i} \hat{S}_{i}^{\top}$, which is a "channel $\times$ channel" matrix (in our setting, it is $49 \times 49$ ). We arrange all $X_{i}, i=1, \ldots, Z$ to form a tensor of dimensions $Z \times 49 \times 49$.

For our experiments, we used $Z=5$ different band-pass Butterworth filters with cutoff frequencies of $(7,10),(9$ 12), (11 14), (13 16) and (15 18) with scaling by 50 which resulted in a signal converted into a tensor of dimensions $5 \times 49 \times 49$. We split the data used in the competition into training and validation sets with proportion of $80: 20$, and 
the rest of the data are used for testing. As in the previous experiment, we used logistic regression with all the tensor norms, individual mode unfolded matrix regularizations, and cross validation with unfolded matrix regularization. We also used vector-based logistic regression with $l_{2}$-regularization for comparison. To compare tensor-based methods with the previously proposed matrix approach (Tomioka and Aihara, 2007), we averaged tensor data over the frequency mode and applied classification with matrix trace norm regularization. For all experiment, we selected all regularization parameters in 100 splits in logarithmic scale from 0.01 to 500 .

The results of the experiment are given in Table 2, which strongly indicate that vectorbased logistic regression is clearly outperformed by the overlapped and scaled latent trace norms. Also, in most cases, the averaged matrix method performs poorly compared to the optimal tensor structured regularization methods. Mode-1 regularization performs poorly since mode- 1 was high ranked compared to the other modes. Similarly, the latent trace norm gives poor performance since it cannot properly regularize since it does not consider the rank relative to the mode dimension. For all subjects, mode- 2 and mode- 3 unfolded regularizations result in the same performance due to the symmetry of each $X_{i}$ resulting in same rank along mode- 2 and mode- 3 unfoldings. For subject $a a$, the scaled latent norm, mode-1, mode-2, and mode-wise cross validation give the best or comparable performance. In subject $a l$, all classifiers except the latent norm and mode-1 regularization gives comparable performance. For all other subjects except for $a a$ and $a l$, the overlapped trace norm gives the best performance.

In contrast to the computation time for regression experiments, in this experiment, we see that the computation time for tensor trace norm regularizations are more expensive compared to the mode-wise regularization. Also, the mode-wise cross validation is computationally less expensive than the scaled latent trace norm and other tensor trace norms. This is a slight drawback with the tensor norms, though they tend to have higher classification accuracy.

\section{Conclusion and Future Work}

In this paper, we have studied tensor-based regression and classification with regularization using the overlapped trace norm, the latent trace norm, and the scaled latent trace norm. We have provided dual optimization methods, theoretical analysis and experimental evaluations to understand tensor-based inductive learning. Our theoretical analysis on excess risk bounds showed the relationship of excess risks with the multilinear ranks and dimensions of the weight tensor. Our experimental results on both simulated and real data sets further confirmed the validity of our theoretical analyses. From the theoretical and empirical results, we can conclude that the performance of regularization with tensor norms depends on the multilinear ranks and mode dimensions, where the latent and scaled latent norms are more robust in tensors with large variations of multilinear ranks.

Our research opens up many future research directions. For example, an important direction is on improvement of optimization methods. Optimization over the latent tensors that results in the use of the latent trace norm and the scaled latent trace norm increases the computational cost compared to the vectorized methods. Also, computing 
Table 2: Classification error of experiments with the BCI competition IVa data set. The boldfaced figures on the columns aa, al, $a v, a w$, and ay indicate comparable accuracies among classifiers after a t-test with significance of 0.05 .

\begin{tabular}{|c|c|c|c|c|c|c|}
\hline Norm & $\begin{array}{c}\text { Subject } \\
a a\end{array}$ & $\begin{array}{c}\text { Subject } \\
\text { al }\end{array}$ & $\begin{array}{c}\text { Subject } \\
a v\end{array}$ & $\begin{array}{c}\text { Subject } \\
a w\end{array}$ & $\begin{array}{c}\text { Subject } \\
a y\end{array}$ & $\begin{array}{l}\text { Avg. Time } \\
\text { (Second) }\end{array}$ \\
\hline Overlapped Trace Norm & $0.2205(0.0139)$ & $0.0178(0.0)$ & $0.3244(0.0132)$ & $0.0603(0.0071)$ & $0.1254(0.0190)$ & $17986(1489)$ \\
\hline Latent Trace Norm & $0.3107(0.0210)$ & $0.0339(0.0056)$ & $0.3735(0.0218)$ & $0.1549(0.0381)$ & $0.4008(0.0)$ & 20021(14024) \\
\hline Scaled Latent Trace Norm & $0.2080(0.0043)$ & $0.0179(0.0)$ & $0.3694(0.0182)$ & $0.0804(0.0)$ & $0.1980(0.0476)$ & $77123(149024)$ \\
\hline Mode-1 & $0.3205(0.0174)$ & $0.0339(0.0056)$ & $0.3739(0.0211)$ & $0.1450(0.0070)$ & $0.4020(0.0038)$ & $5737(3238)$ \\
\hline Mode-2 & $0.2035(0.0124)$ & $0.0285(0.0225)$ & $0.3653(0.0186)$ & $0.0790(0.0042)$ & $0.1794(0.0025)$ & $5195(1446)$ \\
\hline Mode-3 & $0.2035(0.0124)$ & $0.0285(0.0225)$ & $0.3653(0.0186)$ & $0.0790(0.0042)$ & $0.1794(0.0025)$ & $5223(1452)$ \\
\hline Mode-wise CV & $0.2080(0.0369)$ & $0.0428(0.0305)$ & $0.3545(0.01255)$ & $0.1008(0.0227)$ & $0.1452(0.0224)$ & $14473(4142)$ \\
\hline Averaged Matrix & $0.2732(0.0286)$ & $0.0178(0.000)$ & $0.4030(0.2487)$ & $0.1366(0.0056)$ & $0.1825(0.0)$ & $1936(472)$ \\
\hline Logistic regression $\left(l_{2}\right)$ & $0.3161(0.0075)$ & $0.0179(0.0)$ & $0.3684(0.0537)$ & $0.2241(0.0432)$ & $0.4040(0.0640)$ & $72(62)$ \\
\hline
\end{tabular}


multiple singular value decompositions and solving Newton optimization sub-problems (for logistic regression) at each iterative step are computationally expensive. This is evident from our experimental results on computation time for regression and classification. It would be an important direction to develop computationally more efficient methods for learning with tensor data to make it more practical.

Regularization with a mixture of norms is common in both vector-based (e.g., the elastic net (Zou and Hastie, 2003)) and matrix-based regularizations (Savalle et al., 2012). It would be an interesting research direction to combine sparse regularization (the $l_{1}$-norm) to existing tensor norms. There is also a recent research direction to develop new composite norms such the $(k, q)$-trace norm (Richard et al., 2014). Development of composite tensor norms can be useful for inductive tensor learning to obtain sparse and low-rank solutions.

\section{Acknowledgment}

KW acknowledges the Monbukagakusho MEXT Scholarship and KAKENHI 23120004, RT acknowledges XXXXXX, and MS acknowledges the JST CREST program.

\section{Appendix A}

In this appendix, we derive the dual formulation of the latent trace norms. Let us consider a training data set $\left(\mathcal{X}_{i}, y_{i}\right), i=1, \ldots, m$, where $\mathcal{X}_{i} \in \mathbb{R}^{n_{1} \times \cdots \times n_{K}}$. To derive the dual for the latent trace norms, we rewrite the primal for the regression of (15) as

$$
\begin{gathered}
\min _{\mathcal{W}} \sum_{i}^{m} \frac{1}{2}\left(y_{i}-z_{i}\right)^{2}+\lambda \sum_{k=1}^{K}\left\|W_{(k)}^{(k)}\right\|_{\mathrm{tr}} \\
\text { subject to } z_{i}=\left\langle\sum_{k=1}^{K} \mathcal{W}^{(k)}, \mathcal{X}_{i}\right\rangle+b, \quad i=1, \ldots, m .
\end{gathered}
$$


Its Lagrangian can be written by introducing variables $\alpha_{i} \in \mathrm{R}, i=1, \ldots, m$ as

$$
\begin{aligned}
& G(\alpha)=\min _{\mathcal{W}, z_{1}, \cdots, z_{m}} \sum_{i}^{m} \frac{1}{2}\left(y_{i}-z_{i}\right)^{2}+\lambda \sum_{k=1}^{K}\left\|W_{(k)}^{(k)}\right\|_{\text {tr }}+\sum_{i}^{m} \alpha_{i}\left(z_{i}-\left\langle\sum_{k=1}^{K} \mathcal{W}^{(k)}, \mathcal{X}_{i}\right\rangle+b\right) \\
& =\min _{z_{1}, \cdots, z_{m}} \sum_{i}^{m}\left(\frac{1}{2}\left(y_{i}-z_{i}\right)^{2}+\alpha_{i} z_{i}\right)+\min _{b} b \sum_{i}^{m} \alpha_{i} \\
& +\min _{\mathcal{W}} \lambda \sum_{k=1}^{K}\left(\left\|W_{(k)}^{(k)}\right\|_{\mathrm{tr}}-\left\langle\mathcal{W}_{(k)}^{(k)}, \sum_{i}^{m} \alpha_{i} X_{i(k)}\right\rangle\right) \\
& =\sum_{i=1}^{m}\left(-\frac{1}{2} \alpha_{i}^{2}+\alpha_{i} y_{i}\right)+\sum_{k=1}^{K} \begin{cases}0 & \left\|\sum_{i=1}^{m} \alpha_{i} X_{i(k)}\right\|_{\mathrm{op}} \leq \lambda_{k} \\
-\infty & \text { otherwise }\end{cases} \\
& + \begin{cases}0 & \sum_{i=1}^{m} \alpha_{i}=0 \\
-\infty & \text { otherwise }\end{cases} \\
& =\sum_{i=1}^{m}\left(-\frac{1}{2} \alpha_{i}^{2}+\alpha_{i} y_{i}\right)+\sum_{k=1}^{K} \delta_{\lambda_{k}}\left(\sum_{i=1}^{m} \alpha_{i} X_{i(k)}\right)+\delta\left(\sum_{i=1}^{m} \alpha_{i}\right) .
\end{aligned}
$$

Let us introduce auxiliary variables $\mathcal{V}^{(1)}, \ldots, \mathcal{V}^{(K)}$ to remove the coupling between the indicator functions. Then the above dual solutions can be restated as

$$
\begin{gathered}
\min _{\boldsymbol{\alpha}, \mathcal{V}^{(1)}, \cdots, \mathcal{V}^{(K)}} \sum_{i=1}^{m}\left(-\frac{1}{2} \alpha_{i}^{2}+\alpha_{i} y_{i}\right)+\sum_{k=1}^{K} \delta_{\lambda_{k}}\left(V_{(k)}^{(k)}\right) \\
\text { subject to } \mathcal{V}^{(k)}=\sum_{i=1}^{m} \alpha_{i} \mathcal{X}_{i} \quad k=1, \ldots, K, \\
\sum_{i=1}^{m} \alpha_{i}=0 .
\end{gathered}
$$

Similarly, we can derive the dual formulation for logistic regression.

\section{Appendix B}

In this appendix, we prove the theoretical results in Section 4.

Proof of Lemma 1: By using the same approach as the one given in Wimalawarne et al. (2014); Maurer and Pontil (2013), we rewrite

$$
R(\hat{\mathcal{W}})-R\left(\mathcal{W}^{0}\right)=[R(\hat{\mathcal{W}})-\hat{R}(\hat{\mathcal{W}})]+\left[\hat{R}(\hat{\mathcal{W}})-\hat{R}\left(\mathcal{W}^{0}\right)\right]+\left[\hat{R}\left(\mathcal{W}^{0}\right)-R\left(\mathcal{W}^{0}\right)\right] .
$$

The second term is always negative and based on Hoeffding's inequality, with probability $1-\delta / 2$, the third term can be bounded as $\sqrt{\frac{\ln \left(\frac{1}{\delta}\right)}{2 n}}$ :

$$
\begin{aligned}
R(\hat{\mathcal{W}})-R\left(\mathcal{W}^{0}\right) & \leq R(\hat{\mathcal{W}})-\hat{R}(\hat{\mathcal{W}})+\sqrt{\frac{\log \left(\frac{2}{\delta}\right)}{2 m}} \\
& \leq \sup _{\|\mathcal{W}\|_{*} \leq B_{0}}(R(\mathcal{W})-\hat{R}(\mathcal{W}))+\sqrt{\frac{\log \left(\frac{2}{\delta}\right)}{2 m}} .
\end{aligned}
$$


Further applying McDiarmid's inequality, with probability at least $1-\delta$, we get the following following Rademacher complexity:

$$
\mathfrak{R}=\frac{2}{m} \mathbb{E} \sup _{\|\mathcal{W}\|_{\star} \leq B_{0}} \sum_{i=1}^{m} \sigma_{i} l\left(\left\langle\mathcal{W}, \mathcal{X}_{i}\right\rangle, y_{i}\right),
$$

where $\sigma_{i} \in\{-1,1\}$ are Rademacher variables which leads to

$$
\begin{aligned}
R(\hat{\mathcal{W}})-R\left(\mathcal{W}^{0}\right) & \leq \frac{2}{m} \mathbb{E} \sup _{\|\mathcal{W}\|_{\star} \leq B_{0}} \sum_{i=1}^{m} \sigma_{i} l\left(\left\langle\mathcal{W}, \mathcal{X}_{i}\right\rangle, y_{i}\right)+\sqrt{\frac{\log \left(\frac{2}{\delta}\right)}{2 m}} \\
& \leq \frac{2 \Lambda}{m} \mathbb{E} \sup _{\|\mathcal{W}\|_{\star} \leq B_{0}} \sum_{i=1}^{m} \sigma_{i}\left\langle\mathcal{W}, \mathcal{X}_{i}\right\rangle+\sqrt{\frac{\log \left(\frac{2}{\delta}\right)}{2 m}} \\
& =\frac{2 \Lambda}{m} \mathbb{E} \sup _{\|\mathcal{W}\|_{\star} \leq B_{0}}\left\langle\mathcal{W}, \sum_{i=1}^{m} \sigma_{i} \mathcal{X}_{i}\right\rangle+\sqrt{\frac{\log \left(\frac{2}{\delta}\right)}{2 m}} \\
& \leq \frac{2 \Lambda}{m} \mathbb{E} \sup _{\|\mathcal{W}\|_{\star} \leq B_{0}}\|\mathcal{W}\|_{\star}\|\mathcal{M}\|_{\star^{*}}+\sqrt{\frac{\log \left(\frac{2}{\delta}\right)}{2 m}} \text { (Hölder's inequality) } \\
& \leq \frac{2 \Lambda B_{0}}{m} \mathbb{E}\|\mathcal{M}\|_{\star^{*}}+\sqrt{\frac{\log \left(\frac{2}{\delta}\right)}{2 m}} .
\end{aligned}
$$

Proof of Theorem 1: First we bound the data-dependent component of $\mathbb{E}\|\mathcal{M}\|_{\text {overlap* }}$. For this, we use the following duality relationship borrowed from Tomioka and Suzuki (2013):

$$
\|\mathcal{M}\|_{\text {overlap* }}=\inf _{\mathcal{M}^{(1)}+\cdots+\mathcal{M}^{(K)}=\mathcal{M}} \max _{k}\left\|M_{(k)}^{(k)}\right\|_{\text {op }}
$$

Since we can take any $\mathcal{M}^{(k)}$ to equal $\mathcal{M}$, the above norm can be upper bounded as

$$
\|\mathcal{M}\|_{\text {overlap* }} \leq \min _{k}\left\|M_{(k)}\right\|_{\text {op }} .
$$

Furthermore, the expectation of the minimum of $k$ can be upper-bounded by the minimum of the expectation:

$$
\mathbb{E}\|\mathcal{M}\|_{\text {overlap* }} \leq \mathbb{E} \min _{k}\left\|M_{(k)}\right\|_{\text {op }} \leq \min _{k} \mathbb{E}\left\|M_{(k)}\right\|_{\text {op }}
$$

Let $\boldsymbol{\sigma}=\left\{\sigma_{1}, \cdots, \sigma_{m}\right\}$ be fixed Rademacher variables. Since each $\mathcal{X}_{i}$ contains elements following the standard Gaussian distribution, it makes each element in $\mathcal{M}$ a sample from $\mathcal{N}\left(0,\|\boldsymbol{\sigma}\|_{2}^{2}\right)$. Based on the standard methods used in Tomioka et al. (2011b), we can express $\left\|M_{(k)}\right\|_{\text {op }}$ as

$$
\left\|M_{(k)}\right\|_{\text {op }}=\sup _{u \in S^{n_{k}-1}, v \in S^{\Pi_{i \neq k} n_{i}-1}} u^{\top} M_{(k)} v .
$$

Using Gordan's theorem as in Tomioka et al. (2011b), we have

$$
\mathbb{E}\left\|M_{(k)}\right\|_{\text {op }} \leq\|\boldsymbol{\sigma}\| \min _{k}\left(\sqrt{n_{k}}+\sqrt{n_{\backslash k}}\right) .
$$


Next taking the expectation over $\sigma$, we have

$$
\mathbb{E}\|\boldsymbol{\sigma}\|_{2} \leq \sqrt{\mathbb{E}_{\boldsymbol{\sigma}}\|\boldsymbol{\sigma}\|_{2}^{2}}=\sqrt{m}
$$

Combining (201) and (21) with (19) results in

$$
\mathbb{E}\|\mathcal{M}\|_{\text {overlap}^{*}} \leq \min _{k} \sqrt{m}\left(\sqrt{n_{k}}+\sqrt{n_{\backslash k}}\right) .
$$

Finally, the excess loss can be written as

$$
R(\hat{W})-R\left(W^{0}\right) \leq c_{1} \Lambda \frac{B}{\sqrt{m}}\left(\sum_{k=1}^{K} \sqrt{r_{k}}\right) \min _{k}\left(\sqrt{n_{k}}+\sqrt{n_{\backslash k}}\right)+c_{2} \sqrt{\frac{\log \left(\frac{2}{\delta}\right)}{2 m}} .
$$

Proof of Theorem 2 :To bound the data-dependent component, we use the duality result given in Tomioka and Suzuki (2013):

$$
\|\mathcal{M}\|_{\text {latent }}{ }=\max _{k}\left\|M_{(k)}\right\|_{\text {op }}
$$

Since $\mathcal{M}$ consists of elements following the standard Gaussian distribution, for each mode $k$ unfolding, we can write a tail bound (Tomioka and Suzuki, 2013) as

$$
P\left(\left\|M_{(k)}\right\|_{\text {op }} \geq\|\boldsymbol{\sigma}\|\left(\sqrt{n_{k}}+\sqrt{n_{\backslash k}}\right)+t\right) \leq \exp \left(-t^{2} /\left(2 \sigma^{2}\right)\right) .
$$

Using a union bound, we have

$$
P\left(\max _{k}\left\|M_{(k)}\right\|_{\mathrm{op}} \geq\|\boldsymbol{\sigma}\| \max _{k}\left(\sqrt{n_{k}}+\sqrt{n_{\backslash k}}\right)+t\right) \leq K \exp \left(-t^{2} /\left(2 \sigma^{2}\right)\right),
$$

and this results in

$$
\mathbb{E} \max _{k}\left\|M_{(k)}\right\|_{\text {op }} \leq\|\boldsymbol{\sigma}\| \max _{k}\left(\sqrt{n_{k}}+\sqrt{n_{\backslash k}}\right)+\sigma C \sqrt{2 \log (K)},
$$

where $C$ is a constant. Similarly to (21), taking the expectation over $\boldsymbol{\sigma}$, we arrive at

$$
\mathbb{E} \max _{k}\left\|M_{(k)}\right\|_{\text {op }} \leq \sqrt{m} \max _{k}\left(\sqrt{n_{k}}+\sqrt{n_{\backslash k}}\right)+\sqrt{m} C \sqrt{2 \log (K)},
$$

where $C$ is constant. Finally, the excess risk is given as

$$
R(\hat{W})-R\left(W^{0}\right) \leq c_{1} \Lambda B \sqrt{\frac{\min _{k} r_{k}}{m}}\left(\max _{k}\left(\sqrt{n_{k}}+\sqrt{n_{\backslash k}}\right)+C \sqrt{2 \log (K)}\right)+c_{2} \sqrt{\frac{\log \left(\frac{2}{\delta}\right)}{2 m}} .
$$

Proof of Theorem 3: From Tomioka and Suzuki (2013), we have

$$
\|\mathcal{M}\|_{\text {scaled }}{ }^{*} \max _{k} \sqrt{n_{k}}\left\|M_{(k)}\right\|_{\text {op }}
$$

Using a similar approach to the latent trace norm with the additional scaling of $\sqrt{n_{k}}$, we arrive at the following excess bound for the scaled latent trace norm:

$$
R(\hat{W})-R\left(W^{0}\right) \leq c_{1} \Lambda B \sqrt{\frac{1}{m} \min _{k}\left(\frac{r_{k}}{n_{k}}\right)}\left(\max _{k}\left(n_{k}+\sqrt{N}\right)+C \sqrt{2 \log (K)}\right)+c_{2} \sqrt{\frac{\log \left(\frac{2}{\delta}\right)}{2 m}} .
$$




\section{References}

Bahadori, M. T., Yu, Q. R., and Liu, Y. (2014). Fast multivariate spatio-temporal analysis via low rank tensor learning. In Advances in Neural Information Processing Systems 27, pages 3491-3499

Bertsekas, D. P. (1996). Constrained Optimization and Lagrange Multiplier Methods (Optimization and Neural Computation Series). Athena Scientific.

Boyd, S., Parikh, N., Chu, E., Peleato, B., and Eckstein, J. (2011). Distributed optimization and statistical learning via the alternating direction method of multipliers. Foundations and Trends in Machine Learning, 3(1):1-122.

Cai, J., Cands, E. J., and Shen, Z. (2010). A Singular Value Thresholding Algorithm for Matrix Completion. SIAM J. on Optimization 20, 4, 1956-1982.

Candès, E. J., Li, X., Ma, Y., and Wright, J. (2011). Robust principal component analysis? Journal of the ACM, 58(3):1-37.

Dornhege, G., Blankertz, B., Curio, G., and Müller, K.-R. (2004). Boosting bit rates in Noninvasive EEG single-trial classifications by feature combination and multiclass paradigms. IEEE Transactions on Biomedical Engineering, 51(6):993-1002.

Gabay, D. and Mercier, B. (1976). A dual algorithm for the solution of nonlinear variational problems via finite element approximation. Computers 85 Mathematics with Applications, 2(1):17 - 40 .

Gandy, S., Recht, B., and Yamada, I. (2011). Tensor completion and low-n-rank tensor recovery via convex optimization. Inverse Problems, 27(2):025010.

Ganesh, A., Lin, Z., Wright, J., Wu, L., Chen, M., and Ma, Y. (2009). Fast convex optimization algorithms for exact recovery of a corrupted low-rank matrix. In Proceedings of 3rd IEEE International Workshop on Computational Advances in Multi-Sensor Adaptive Processing, pages 213-216.

Karatzoglou, A., Amatriain, X., Baltrunas, L., and Oliver, N. (2010). Multiverse recommendation: N-dimensional tensor factorization for context-aware collaborative filtering. In Proceedings of the Fourth ACM Conference on Recommender Systems, pages 79-86.

Kolda, T. G. and Bader, B. W. (2009). Tensor decompositions and applications. SIAM Review, 51(3):455-500.

Kim, T.-K., Wong, S.-F., and Cipolla, R. (2007). Tensor canonical correlation analysis for action classification. In Proceedings of the IEEE Conference on Computer Vision and Pattern Recognition, pages 1-8.

Liu, G., Lin, Z., and Yu, Y. (2010). Robust subspace segmentation by low-rank representation. In Proceedings of the 27th International Conference on Machine Learning (ICML-10), pages 663-670. 
Liu, J., Musialski, P., Wonka, P., and Ye, J. (2009). Tensor completion for estimating missing values in visual data. In Proceedings of the IEEE International Conference on Computer Vision, pages 2114-2121.

Maurer, A. and Pontil, M. (2013). Excess risk bounds for multitask learning with trace norm regularization. In Proceedings of the Annual Conference on Learning Theory 2013, pages 55-76.

Recht, B., Fazel, M., and Parrilo, P. (2010). Guaranteed minimum-rank solutions of linear matrix equations via nuclear norm minimization. SIAM Review, 52(3):471-501.

Richard, E., Obozinski, G. R., and Vert, J.-P. (2014). Tight convex relaxations for sparse matrix factorization. In Advances in Neural Information Processing Systems 27, pages $3284-3292$.

Romera-Paredes, B., Aung, H., Bianchi-Berthouze, N., and Pontil, M. (2013). Multilinear multitask learning. In Proceedings of the 30th International Conference on Machine Learning, pages 1444-1452.

Sankaranarayanan, P., Schomay, T. E., Aiello, K. A., and Alter, O. (2015). Tensor GSVD of patient- and platform-matched tumor and normal DNA copy-number profiles uncovers chromosome arm-wide patterns of tumor-exclusive platform-consistent alterations encoding for cell transformation and predicting ovarian cancer survival. PLoS ONE, 10(4):e0121396.

Savalle, P., Richard, E., and Vayatis, N. (2012). Estimation of simultaneously sparse and low rank matrices. In Proceedings of the 29th International Conference on Machine Learning, pages 1351-1358.

Signoretto, M., Dinh, Q. T., De Lathauwer, L., and Suykens, J. A. K. (2013). Learning with tensors: a framework based on convex optimization and spectral regularization. Machine Learning, 94(3):303-351.

Tomioka, R. and Aihara, K. (2007). Classifying matrices with a spectral regularization. In Proceedings of International Conference on Machine Learning, pages 895-902.

Tomioka, R., Hayashi, K., and Kashima, H. (2011a). Estimation of low-rank tensors via convex optimization. Technical report, arXiv 1010.0789.

Tomioka, R. and Suzuki, T. (2013). Convex tensor decomposition via structured Schatten norm regularization. In Advances in Neural Information Processing Systems 26, pages $1331-1339$.

Tomioka, R., Suzuki, T., Hayashi, K., and Kashima, H. (2011b). Statistical performance of convex tensor decomposition. In Advances in Neural Information Processing Systems 24, pages $972-980$.

Tomioka, R., Suzuki, T., and Sugiyama, M. (2011c). Super-linear convergence of dual augmented-Lagrangian algorithm for sparsity regularized estimation. Journal of $M a-$ chine Learning Research, 12(May):1537-1586, 2011. 
Wimalawarne, K., Sugiyama, M., and Tomioka, R. (2014). Multitask learning meets tensor factorization: task imputation via convex optimization. In Advances in Neural Information Processing Systems 27, pages 2825-2833.

Zhou, H. and Li, L. (2014). Regularized matrix regression. Journal of the Royal Statistical Society: Series B (Statistical Methodology), 76(2):463-483.

Zhou, H., Li, L., and Zhu, H. (2013). Tensor regression with applications in neuroimaging data analysis. Journal of the American Statistical Association, 108(502):540-552.

Zou, H. and Hastie, T. (2003). Regularization and Variable Selection via the Elastic Net. Journal of the Royal Statistical Society: Series B (Statistical Methodology), 67(2):301320. 


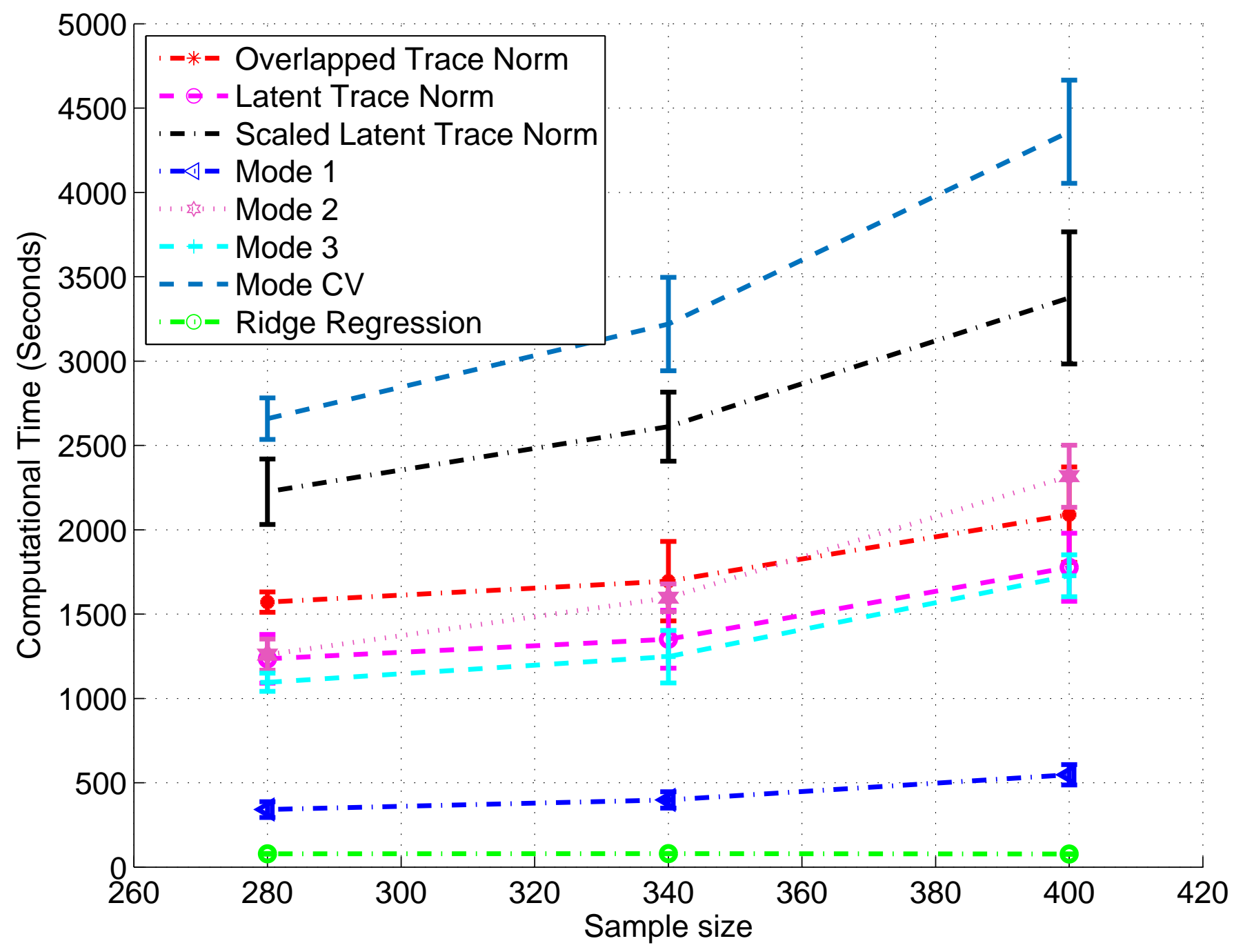

